\title{
Brief Study of Identification System Using Regression Neural Network Based on Delay Tap Structures
}

\author{
Alvin Sahroni
}

\begin{abstract}
- this investigation aims to provide an overview and investigations of non-linear identification system using neural network approach. Nowadays, a lot of neural network approach was done to provide a satisfying identification system. Backpropagation scheme as the mainstream approach for developing identification system has several limitations such as training data, computation time, architecture, optimization technique for weight value update, and many others. Regression Neural Network which is found by specht on 1990 contains more advantages compare with backpropagation scheme. With the improvement of computation time, architecture, and robustness of this model and provided $90 \%$ of effectiveness, promising a good prospective to develop non-linear identification system. For future works, it can be implemented in neural network predictive model control system and another control scheme based on identification system approach.
\end{abstract}

Keywords-GRNN, Identification System, backpropagation, predictive model

\section{INTRODUCTION}

$\mathrm{T}$ HE development of neural networks has been started since several decades ago. Since the past decade, many artificial intelligence methods such as fuzzy logic, neural networks, etc. has been developed to solving system modeling for dynamic plants. For nonlinear problems, neural networks have been widely applied for empirical process modeling [1]. The development of Neural networks itself increase rapidly since the multi layer neural networks and backpropagation was founded and popular related to the its capability to be trained to associate input data to output data that can be useful to learn unknown dynamic plants. The popularity of this scheme have been used in complex identification system [2]. Currently, neural networks are being applied to physical plant modelling with satisfactory results $[3,4]$.

System modelling and identification system was applied in control system engineering. In control engineering, neural network approach based modelling are generally used as dynamic plant emulators for doing control design and also can

Manuscript received September 13th, 2013. This work was supported by Electrical Engineering Department of UII Yogyakarta, Indonesia. Independent Research Project in Control and Microprocessor Laboratory, UII Indonesia. Alvin Sahroni is with the Department of Electrical Engineering, UII Yogyakarta Indonesia, Kaliurang Street 14.5 (alvinsahroni@uii.ac.id). be utilize for predictor models in many adaptive control configurations $[5,6,7,8,9]$. Many of controller using direct controllers scheme, but in neural networks as direct controllers to the plant are less popular, however several methos have been reported. The common and best schemes of neural networks controllers are using predictor model in a control system which used to estimate the future condition/value. The problem came related to the implementation of predictor model that usulally has constraints of limited network size, and in many real time applications, fast computation time and processing is required.

Based on the problems, using conventional multilayer and backpropagation in complex system give lacks of system performance. And in reality conditions, many kinds of identification and control configurations applied online schemes which needs fast and continuous learning capability and large of data to be trained. In many kinds of neural networks paradigms, the General Regression Neural Networks (GRNN) seems to be promising to posses such as characteristics [10] thus, it's being used as the motivation for this investigation.

GRNN has been applied in many number of applications on identification/modelling system and control [11]. There also have been investigated certain comparison studies to demonstrate the modelling capability of GRNN compare with another multilayer and common neural networks paradigms $[12,13]$. The investigation results gives advantages by applying GRNN than using backpropagation paradigms. The significant result related to the computation time, neural networks architectures, and simplify of neural network algorithms have been provided. This investigation also prove the advantages of GRNN based results and performances. Another investigation also reported using hybrid method between GRNN and fuzzy clustering measurement in system identification. The work also related to the simplified GRNN methods to provide a good performance using large of training data [. This investigation itsetlf will provided several configuration of delay tap/time to increase the performance of GRNN in system identification.

This paper has several parts that organized as follows, A brief introduction and background review of identification system using neural networks and GRNN which given in this part. It's followed by explanation of GRNN, and identification 
system applied in dynamic plants. The next part will provide proposed method of identification system using GRNN on dynamic plants. The proposed method that has been investigated will be discussed on the next part and also closed by doing conclusion for certain investigations.

\section{TRAINING OF GRNN}

The basic fundamental of GRNN has been proposed [10] (Fig.1) by specht and the using of GRNN has been popular than back-propagation because the characteristic of GRNN as probabilistic solution and the known as probabilistic neural network with fast computation and training process and its simplicity [15], then GRNN was the alternative feed-forward method after back-propagation in many fields.

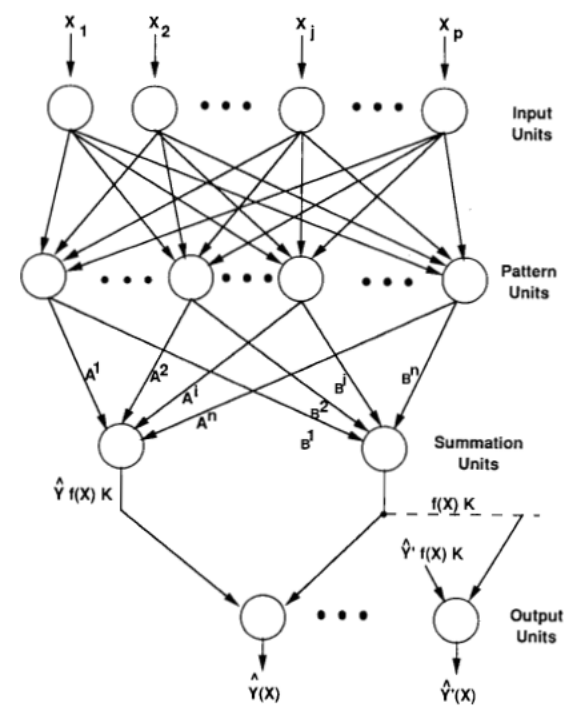

Figure 1. GRNN Architecture (Specht, 1991)

GRNN has two layers, the first layer called Radial Basis Layer and the second layer called as Linearization Layer or Special Linear Layer (Fig.2). Where $\mathrm{R}=$ number elements in input vector, $\mathrm{Q}=$ number of neurons.

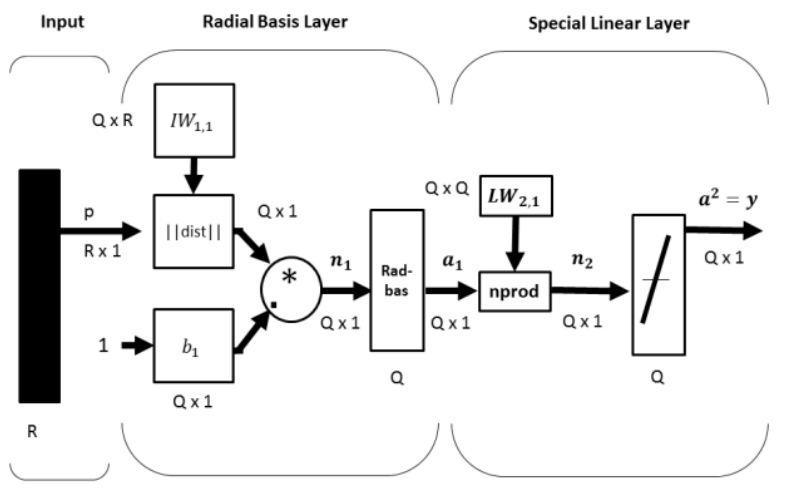

Figure 2. GRNN Architecture

The concept is the regression function performed on an independent variable such as $X$ computes the most probable value of dependent variable $Y$ within finite set of observations data. Let's $f(x)$ as the probability density function of a random vector $x$. Regression result of $Y$ given by $X$ based on below formula:

$$
E[y / X]=\frac{\int_{-\infty}^{\infty} y \cdot f(X, y) d y}{\int_{-\infty}^{\infty} f(X, y) d y}
$$

The probability density function must be estimated from sample observations (data sets) of $x$ and $y$. The general form of estimation process given by:

$$
f_{n}(x)=\frac{1}{n \lambda} \sum_{i=1}^{n} \varphi\left(\frac{x-x_{i}}{\sigma}\right)
$$

Where $x_{i}$ are independent and as distributed random variables with absolutely continuous distribution function. The $\varphi$ have constrains to bounds certain value to satisfy several conditions:

$$
\begin{aligned}
& \int_{-\infty}^{+\infty}|\varphi(y)| d y<\infty \\
& \lim _{y \rightarrow \infty}|y \varphi(y)|=0
\end{aligned}
$$

And

$$
\int_{-\infty}^{+\infty} \varphi(y) d y=1
$$

The function $\sigma=\sigma(n)$ must be chosen with:

$$
\begin{aligned}
& \lim _{n \rightarrow \infty} \sigma(n)=0 \\
& \lim _{n \rightarrow \infty} n \sigma^{2}(n)=\infty
\end{aligned}
$$

One useful form of the weighting function $\varphi$ is the kernel density function which is known as Gaussian function. There is a constant value for distribution estimator that based on panzer's results that will converge to the underlying distribution at the sample point when it is smooth and continuous. Based upon sample values $X^{i}$ and $Y^{i}$ of the random variable $x$ and $y$, it can provide a good distribution constant value as given by [9]:

$$
\begin{aligned}
& \hat{f}(X, Y)=\frac{1}{(2 \pi)^{(p+1) / 2} \sigma^{(p+1)}} . \\
& \frac{1}{n} \sum_{i=1}^{n} e^{\left[-\frac{\left(X-X^{i}\right)^{T}\left(X-X^{i}\right)}{2 \sigma^{2}}\right]} e^{\left[-\frac{\left(Y-Y^{i}\right)^{2}}{2 \sigma^{2}}\right]}
\end{aligned}
$$

$p$ is the dimension of the vector variable $x, n$ is the number of observations, $\sigma$ is the width spreading of 
estimated kernel or smoothing factor, and $Y^{i}$ is the desired or target data given by the observed input data of $X^{i}$. Then define $D_{i}^{2}$ by using equation:

$$
D_{i}^{2}=\left(X-X^{i}\right)^{T}\left(X-X^{i}\right)
$$

Combining (8) and (9) and interchanging the order of integration and summation and given by:

$$
\hat{Y}(X)=\frac{\sum_{i=1}^{N} Y^{i} e^{\left(-\frac{D_{i}^{2}}{2 \sigma^{2}}\right)}}{\sum_{i=1}^{N} e^{\left(-\frac{D_{i}^{2}}{2 \varsigma^{2}}\right)}}
$$

The $Y(X)$ result can be used as weighted average of all observed data of $Y^{i}$ and each observed data is based on weighted exponentially of related to Euclidean distance from $X$. The main difference between probabilistic neural networks and backpropagation scheme within training process is the variables characteristic scheme.

The GRNN paradigms also depend on the decision of best training related to the value of $\sigma$ which can be applied between $0-1$ values and should not be 0 value. The highest value will give good fitting curve and best generalization results. Otherwise, the lower value $\sigma$ will give best fitting curve but will decrease the capability of generalization (Fig.3).

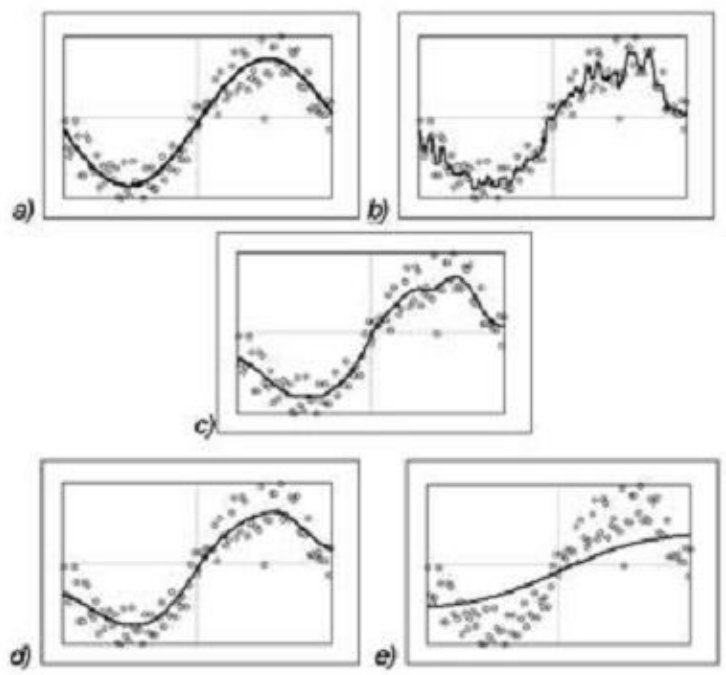

Figure 3. A Simple problem to illustrate the influence of $\sigma$ parameter on the GRNN result a)true function, b)too small, c)average, d)a perfect parameter, e)too large (Vadim

Timonin, et al., 2005[15])

\section{PROPOSED METHOD}

The investigation contains several steps. The steps should be accomplished to provide desired value based from training data sets. There are several schemes that can be applied for identification system based on neural networks paradigms. This investigation will using common scheme of system modeling around neural networks application (Fig. 4).

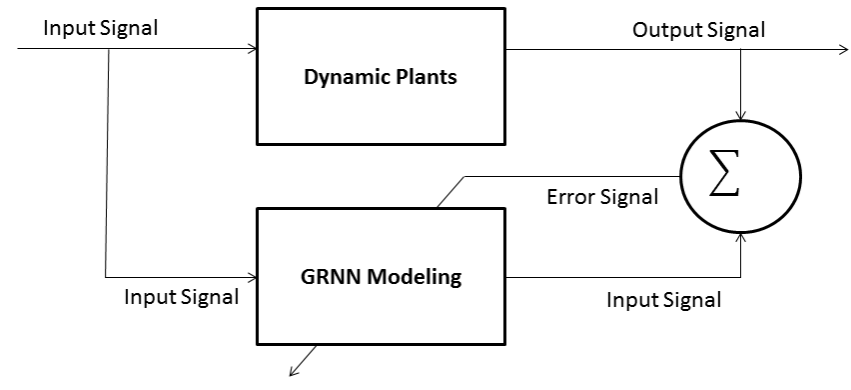

Figure 4. GRNN Scheme

Step 1 : create a GRNN architecture with delay tap/time configurations

Step 2 : Testing and validating the architecture/models and calculate MSE(eq.11) value

$$
M S E=\frac{1}{n} \sum_{i=1}^{n}\left(y_{(i) \text { real }}-y_{(i) \text { predict }}\right)
$$

Step 3 : if the MSE of fitting curve is larger than desired value, develop a new scenarios and back to Step 2.

Step 4 : After providing best architecture, it will conclude the best identification system for the problem

As the title above, this investigation will focused on using delay tapping/time configurations to provide a good architecture of GRNN's performance. Configuration itself is how we can design certain delay tapping for input and output layer of GRNN to gain best fit of dynamic plant identification system (Fig 5.).

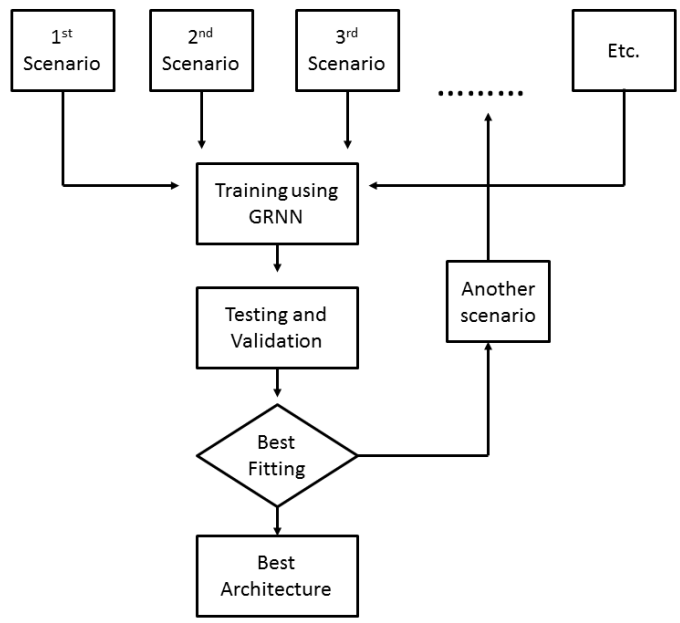

Figure 5. Step for the proposed method

MSE performance will be the main parameters to measure the feasibility of each scenario (Table 1.). Each scenario will provide performance information of each architecture scenario. This investigation will provide the best 
architecture of GRNN using delay tap/time configuration.

TABLE 1.

SEQUENCE TO INVESTIGATE SEVERAL SCENARIO OF DELAY TAPPING/TIME FOR IDENTIFICATION SYSTEM USING GRNN

\begin{tabular}{ccc} 
No & Scenarios & Performance \\
\hline 1 & $1^{\text {st }}$ scenario & \\
2 & $2^{\text {nd }}$ scenario & \\
3 & $3^{\text {rd }}$ scenario & \\
4 & $\ldots$ etc. & \\
\hline
\end{tabular}

\section{ANALYSIS AND DISCUSSION}

The investigation will using hair dryer for the objects which will identified the system. Hair dryer is a device that processed air fanned through a tube and heated at the inlet. The air temperature is measure by a thermocouple at the outlet. The input $u(k)$ is the voltage over a mesh of resistor wire to heat incoming air; the output $y(k)$ is the outlet air temperature (Fig.6.).

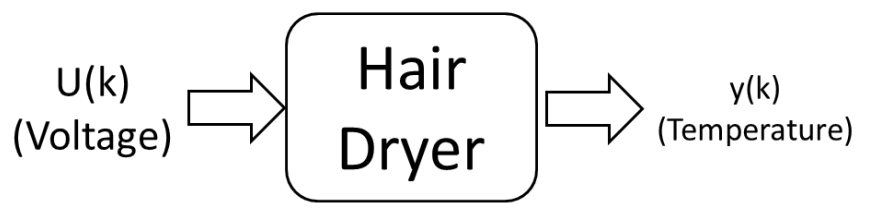

Figure 6. Hair Dryer System
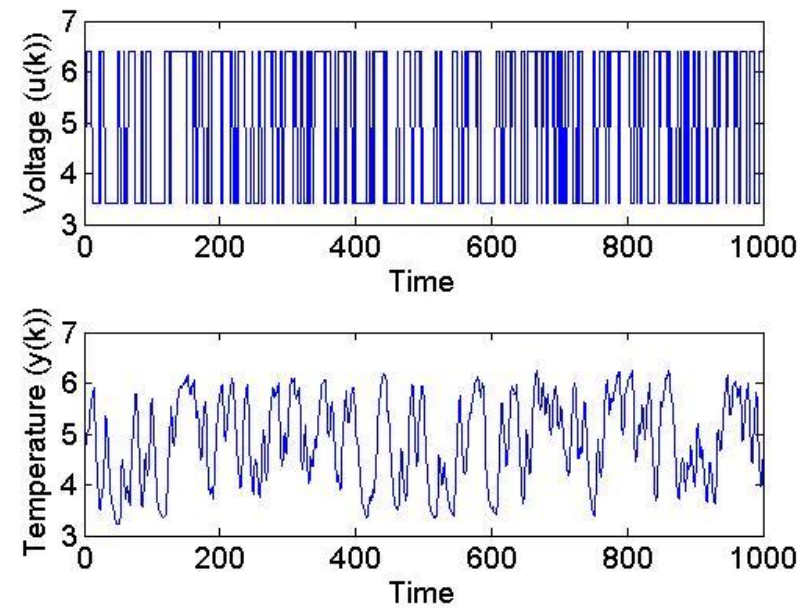

Figure 7. Relationship between $u(k)$ and $y(k)$

The relationship of input $u(k)$ and output $y(k)$ that seen in (Fig.7) conclude that the nonlinear characteristic is provided by this system. The data named as training data will be divided into two parts, $50 \%$ data for modeling/identification system, and $50 \%$ data for validation test (Fig. 8 and Fig. 9) from total data set.
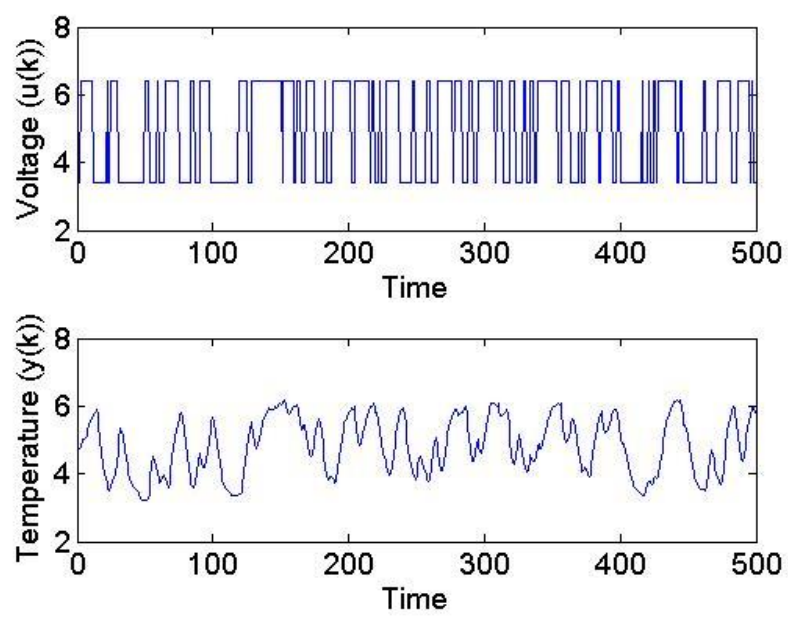

Figure 8. Training data set
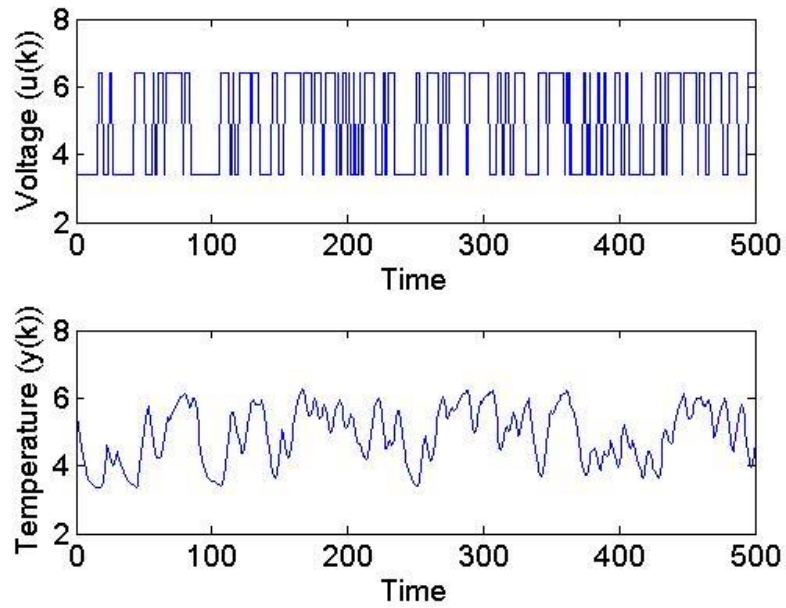

Figure 9. Validation data set

GRNN architecture will conduct several configurations to find the best architecture to identify hair dryer dynamic plant (Table 2.). Configurations of GRNN will be focused on to find the best scheme using delay tap/tapping as the input of dynamic plant. For each delay tap/time, it will be measured by using MSE value to compare between real data and validation test (eq.12).

$y_{(k+1)}=f\left(u_{(k-0)}, u_{(k-1)}, . u_{(k-n)}, y_{(k-0)}, y_{(k-1)}, . . y_{(k-n)}\right)$

Since $u_{(k-n)}$ was the input that belongs to the actuator value of voltage to hair dryer dynamic plant, and $y_{(k-n)}$ as the output sequence of process value. The strategy is how to develop GRNN architectures by combining them, it will provide a good quality of identification non-linear dynamic plants. The measurements of MSE will be indicating the best architecture of GRNN as the identification system for dynamic plants. In the previous section, it has been discussed about spreading coefficent $(\sigma)$. This investigation will be fixed parameters of $\sigma$, based on previous investigation that the higher value of 
$\sigma$ will not promise a good performance. Apparently, to generalize the predicted output, it should been done fairly by giving halfway value (between 0.5-0.9). Indeed that the maximum value of $\sigma$ will give lack of performance within validation phase. But, in the other case, reducing $\sigma$ will improve the training performance phase, but lack of capability to generalize predicting phase. Therefore, by choosing $\sigma$ fixed value that related to previous investigation will help this investigation to configure the best delay tap/time on GRNN architecture. This investigation will use $\sigma=0.8$.

TABLE 2.

SCENARIOS

\begin{tabular}{cc} 
No & Scenarios \\
\hline 1 & 10 input $\left(y_{(k-1) . .(k-4)}, u_{(k-1) . .(k-4)}\right)$ \\
2 & 5 input $\left(y_{(k-1) . .(k-2)}, u_{(k-1) . .(k-3)}\right)$ \\
3 & 3 input $\left(y_{(k-1) . .(k-2)}, u_{(k-1)}\right)$ \\
4 & 2 input $\left(y_{(k-1)}, u_{(k-1)}\right)$ \\
5 & 8 input $\left(y_{(k-1) . .(k-4)}, u_{(k-1) . .(k-4)}\right)$ \\
6 & 12 input $\left(y_{(k-1) . .(k-8)}, u_{(k-1) . .(k-4)}\right)$ \\
\hline
\end{tabular}

\section{CONCLUSION}

The investigation has provided several conclusions that GRNN as the identifications tool has a feasibility performance and shown good performance. The identification of the system depends on input characterization. The characterization refers to delay tap/time configuration within GRNN architecture. The performance affected by two parameters. Spreading coefficients ( $\sigma$ ) will give better result based on generalization using a higher value (between $0.5-0.9$ ). In training phase, a higher value of $\sigma$ will not give a best fitting performance. Otherwise, using lower value of $\sigma$ affected to the smooth fitting curve performance in training performance and gives a lack of generalization capability. Other parameters that affected the performance of GRNN in the identification problem is while choosing architecture for GRNN input include delay tap/time configuration as the input neuron. While increasing the amount of neuron indicating delay tap/time, cannot be surely as the most optimize result of identification dynamic system. For future works, it will compare with statistical model for dynamic plants such as ARX and ARMAX identification based system.

\section{REFERENCES}

[1] Teo Lian Seng, Marzuki Khalid, and Rubiyah Yusof, "Adaptive GRNN Modelling of Dynamic Plants," in Proceedings of the 2002 IEEE International Symposium on Intelligent Control, Canada, 2002, pp. 217222.

[2] B. Kosko, Neural Network and Fuzzy Systems. USA: Prentice Hall, 1992.

[3] Pao YH, SM Phillips, and Sobajic D.J, "Neural-net computing and the intelligent control of systems," International Journal Control, pp. 263289, 1992.

[4] D. Gorinevsky, "On the persistency of excitation in radial basis function network identification of nonlinear systems," IEEE trans. on neural networks, pp. 1237-1244, 1995.

[5] G Irwin , M Brown, Hogg B, and E Swidenbank, "Neural Network modelling of a 200MW Boiler System," IEEE Proc. Control Theory Applications Vol : 142, pp. 529-536, 1995.

[6] A Draeger, S Engell, and H Ranke, "Model Predictive Control Using Neural Network," IEEE COntrol Sys. Mag Vol : 15, pp. 61-66, 1995.

[7] S Omatu, M Khalid, and R Yusof, Neuro-Control and Its Application. London: Springer-Verlag, 1995.

[8] M Smith, Neural Networks for Statistical Modelling. USA: Van Nonstrand Reinhold, 1993.

[9] K.J hunt and D Sbarbaro, "Adaptive Filtering and Neural Networks for realisation of internal model control," Intelligent System Engineering, pp. 67-75, 1993.

[10] D.F Specht, "Probabilistic and General Regression Neural Network," in Fuzzy logic and Neural Network Handbook.: McGraw-Hill Companies, Inc, 1996.

[11 B.G. Hyun and K. Nam, "Fault Diagnoses of Rotating Machines by ] Using Neural Networks:GRNN and BPN," in Proceeding of the 1995 IEEE IECON, 1995, pp. 1456-1461.

[12] L. Marquez and T. Hill, "Function Approximation Using Backpropagation and General Regression Neural Networks," in Proc. 26th Hawaii International Conf. on System Sciences, Hawaii, 1995, pp. 1422-1427.

[13] Alvin Sahroni, "Time Series Prediction Comparison Study based on General Regression Neural Network (GRNN) and Backpropagation Neural Network in Electricity Peak Load Prediction in Indonesia ," in TEKNOIN 2011, Yogyakarta Indonesia, 2011.

[14] Shi-jun Zhao, Jin-Lei ZHang, Xunli, and Wei Song, "A Generalized Regression Neural Network Based on Fuzzy Means Clustering and Its Application in System Identification," in Information Technology Convergence, 2007. ISITC 2007. International Symposium on, Joenju, 2007, pp. 13 - 16.

[15] Vadim Timonin and Elena Savelieva, "Spatial Prediction of Radioactivity Using General Regression Neural Network," Monash University EPRESS, Australia, Applied GIS Vol 1 No 2, 2005.

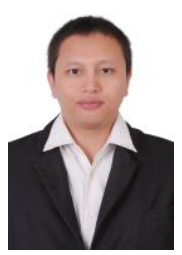

Alvin Sahroni received his bachelor's degree from Universitas Islam Indonesia Yogyakarta, Indonesia, and master's degree from joint program in system engineering between Gadjah Mada University (UGM) -Institut Teknologi Bandung (ITB)-Karlsruhe Institute Tech (KIT, Germany). Now he is currently a full lecturer, researcher, and consultant engineering at Universitas Islam Indonesia Yogyakarta. His current research is in control system, intelligent system, machine learning, and artificial intelligence. His current research is related to the development of neural network and its hybrid system fundamental and applications in several applications. His also planning take his doctoral degree in artificial intelligence system application. 